\title{
Die Bestimmung von Calcium, Strontium und Baryum als Oxalate.
}

\author{
Von \\ Chardes A. Peters. ${ }^{1}$
}

In einer früheren Mitteilung aus diesem Laboratorium ${ }^{2}$ wurden die Versuchsbedingungen beschrieben, unter denen es möglich ist Oxalsäure in Gegenwart von Salzsäure titrimetrisch mit Permanganat zu bestimmen; es wurde damals gefunden, dals der Mehrverbrauch an Permanganat, der gewöhnlich zu konstatieren ist, wenn man diese Titration in der üblichen Weise ausführt, vermieden werden kann, wenn man vor der Titration der Oxalsäure Manganosalz hinzufügt. Diese Thatsache führte zu dem Versuche, die Lösung der Erdalkalioxalate mit Salzsäure zu bewirken, und die freie Oxalsäure mit Permanganat in Gegenwart von Manganosalz zu titrieren; weiterhin wurde hierdurch veranlalst das Studium der Versuchsbedingungen, unter denen die Niederschläge von Baryum- und Strontiumoxalat in einer für quantitative Zwecke hinreichenden Vollständigkeit ausgefällt werden könnten. Die entsprechenden Verhältnisse für das Calciumoxalat sind bereits ermittelt worden.

Der Titer der Permanganatlösung wurde mit frisch umkrystallisiertem Ammonoxalat und mit Oxalsäure eingestellt; beide $\mathrm{Be}$ stimmungen ergaben das gleiche Resultat.

\section{Calciumoxalat.}

Es ist bekannt, dafs man das Calcium bestimmen kann, indem man das ausgefällte Calciumoxalat mit Schwefelsäure behandelt und die frei gemachte Oxalsäure mit Permanganat titriert. ${ }^{3}$ Bei der

\footnotetext{
1 Ins Deutsche äbertragen von J. Kopper.

2 Gooch und Peters, Am. Journ. So. (Sill.) 7, 466.

3 Moнr, Titriermethode, 6. Aufl., S. 227.

z. anorg. Chem. XXIX.
} 
vorliegenden Untersuchung wurde der Calciumoxalatniederschlag in Salzsäure gelöst, und die Oxalsäure in Gegenwart von Manganosalz mit Permanganat titriert. Die Methode wird folgendermalsen ausgeführt: Man fällt die kochend heifse Lösung von Calciumchlorid mit Ammonoxalat, lälst sie 12 Stunden lang stehen und dekantiert die Flüssigkeit über Asbest. Den Niederschlag wäscht man zweibis dreimal durch Dekantieren mit $50-100 \mathrm{ccm}$ kaltem Wasser aus und bringt ihn dann auf das Filter. Hierauf wird der Tiegel mit Inbalt in das zur Fällung benutzte Becherglas zurückgebracht, mit $100-200 \mathrm{ccm}$ Wasser und 5-10 ccm konzentrierter Salzsäure übergossen, und mit $0.5-1.0 \mathrm{~g}$ Manganochlorid versetzt, worauf man die Oxalsäure bei $35-45^{\circ}$ titriert. Die in Tabelle I zusammengestellten Analysenwerte sind, wie man sieht, ausgezeichnet; sie zeigen, dafs man das Calcium als Oxalat in Gegenwart von Chlorwasserstoffsäure und Manganosalz durch Permanganat quantitativ bestimmen kann.

Langandauerndes Waschen mit heifsem Wasser ist zu vermeiden, wenn das zur Fällung verwendete Ammonoxalat völlig entfernt ist. Bei einem Versuche z. B., bei dem der Niederschlag auf dem Asbest $14 \mathrm{mal}$ mit je $50 \mathrm{ccm}$ heilsem Wasser gewaschen worden war, wurden durch diese Menge Waschwasser je 2-6 Tropfen einer annähernd $1 / 10^{-n}$. Permangauatlösung entfärbt, so dafs im ganzen ein Verlust von $0.0034 \mathrm{~g} \mathrm{CaO}$ stattfand.

Tabelle I.

\begin{tabular}{|c|c|c|c|c|}
\hline $\begin{array}{c}\mathrm{CaO} \text {, angew. } \\
\text { als } \mathrm{CaCl}_{2} \\
\mathrm{~g}\end{array}$ & $\begin{array}{c}\text { Ammon- } \\
\text { oxalat } \\
\mathrm{g}\end{array}$ & $\begin{array}{l}\text { Vol. bei der } \\
\text { Fällung } \\
\text { cem }\end{array}$ & $\begin{array}{c}\text { Gefunden } \\
\mathrm{CaO} \\
\mathrm{g}\end{array}$ & $\begin{array}{c}\text { Fehler } \\
\qquad g\end{array}$ \\
\hline 0.0656 & 0.3 & 100 & 0.0657 & +0.0001 \\
\hline 0.0656 & 0.3 & 100 & 0.0656 & 00000 \\
\hline 0.0656 & 0.3 & 150 & 0.0658 & +0.0002 \\
\hline 0.0656 & 0.3 & 100 & 0.0655 & -0.0001 \\
\hline 0.0985 & 0.5 & 175 & 0.0981 & -0.0004 \\
\hline 0.1313 & 0.6 & 150 & 0.1315 & +0.0002 \\
\hline 0.1313 & 0.6 & 200 & 0.1315 & +0.0002 \\
\hline
\end{tabular}

Strontiumoxalat.

Sovchay und Lenssen ${ }^{1}$ haben festgestellt, dafs Strontiumoxalat in 12000 Teilen Wasser löslich ist. Diese Thatsache erschien ein

${ }^{1}$ Lieb. Ann. 102, 35. 
erfolgreiches Studium der quantitativen Abscheidung des Strontiums zu verbürgen. Das Strontiumoxalat wurde in der folgenden Untersuchung sowohl in alkoholischer als auch in wässeriger Lösung ausgefällt; beide Fälle sollen gesondert besprochen werden. Der Gehalt der Strontiumsalzlösungen - deren Reinheit geprüft war wurde festgestellt durch Fällung mit Schwefelsäure aus einer Lösung, die wenigstens zur Hälfte ihres Volumens aus Alkohol bestand; bei einigen Lösungen wurde zur Kontrolle der Gehalt durch Abdampfen mit Schwefelsäure bestimmt.

Fällung in alkoholhaltiger Lösung. Um festzustellen, ob die Fällung des Oxalats in alkoholhaltiger Lösung quantitativ wäre, wurde Strontiumnitrat durch Ammonoxalat aus einer Lösung, die ein Drittel ihres Volumens an Alkohol enthielt, ausgefällt; die Flüssigkeit blieb über Nacht stehen; sodann wurde sie über Asbest filtriert und der Niederschlag wurde in dem mit Untersatz versehenen Filtertiegel mit Schwefelsäure behandelt, geglüht und als Sulfat gewogen.

Die Resultate sind in Tabelle II zusammengestellt.

Tabelle II.

\begin{tabular}{c|c|c|c|c|c}
\hline $\begin{array}{c}\text { SrO, angew. } \\
\text { als Sr(NO } \text {. }_{2}\end{array}$ & $\begin{array}{c}\text { Ammon. } \\
\text { oxalat } \\
\mathrm{g}\end{array}$ & $\begin{array}{c}\text { Vol. bei der } \\
\text { Fällung } \\
\text { ccm }\end{array}$ & $\begin{array}{c}\text { Vol. des } \\
\text { Alkohols }\end{array}$ & $\begin{array}{c}\text { SrO, gef. } \\
\text { als SrSO } \\
\mathrm{g}\end{array}$ & $\begin{array}{c}\text { Differenz } \\
\mathrm{g}\end{array}$ \\
\hline & & & $1 / 3$ & 0.2440 & +0.0006 \\
0.2434 & 0.8 & 180 & $1 / 3$ & 0.2437 & +0.0003 \\
0.2434 & 0.8 & 180 & $1 / 3$ & 0.0022 & 0.0000 \\
0.0022 & 0.2 & 100 & $1 / 3$ & 0.0014 & +0.0001 \\
0.0013 & 0.2 & 100 & $1 / 3$ & 0.0004 & 0.0000 \\
0.0004 & 0.04 & 100 & & &
\end{tabular}

Es geht aus dieser Zusammenstellung hervor, dafs die Fällung auch geringer Mengen von Strontiumsalzen aus einer Lösung, die ein Drittel ihres Volumens an Alkohol enthält, praktisch vollständig ist.

Um die für eine quantitative Fällung des Strontiumoxalats erforderliche Minimalmenge von Alkohol zu bestimmen, wurden einige Versuche ausgeführt, bei denen verschiedene Mengen Alkohol von $85 \%$, sowie verschiedene Mengen Ammonoxalat zur Verwendung gelangten; die Filtrate der so ausgefällten Niederschläge wurden durch Zusatz von mehr Alkohol auf Strontium geprüft.

Die Resultate sind in Tabelle III zusammengestellt. 
Tabelle III.

\begin{tabular}{c|c|c|c|c}
\hline \hline $\begin{array}{c}\mathrm{SrO} \text {, angew. } \\
\left.\text { als } \mathrm{Sr} \mathrm{NO}_{3}\right)_{2} \\
\mathrm{~g}\end{array}$ & $\begin{array}{c}\text { Ammon- } \\
\text { oxalat } \\
\mathrm{g}\end{array}$ & $\begin{array}{c}\text { Vol. der } \\
\text { Flüssigkeit } \\
\mathrm{ecm}\end{array}$ & $\begin{array}{c}\text { Zugesetzte } \\
\text { Menge } \\
\text { \%igen Alkohols }\end{array}$ & $\begin{array}{c}\text { SrO in Filtrat, } \\
\text { gewogen als SrSO } \\
\mathrm{g}\end{array}$ \\
\hline \hline 0.1 & 0.4 & 100 & $1 / 5$ & 0.0000 \\
0.1 & 0.4 & 100 & $1 / 50$ & 0.0000 \\
0.1 & 0.4 & 100 & $1 / 20$ & 0.0004 \\
0.1 & 0.2 & 100 & $1 / 5$ & 0.0000 \\
0.1 & 0.2 & 100 & $1 / 10$ & 0.0009 \\
0.1 & 0.2 & 100 & $1 / 20$ & 0.0020 \\
0.1 & 0.1 & 100 & $1 / 3$ & 0.0002
\end{tabular}

Aus diesen Resultaten geht bervor, dafs bereits bei einem mäfsigen Überschusse von Ammonoxalat ein Fünftel des Gesamtvolumens an $85 \%$ igem Alkohol genügt, die Vollständigkeit der Strontiumoxalatfällung zu bewirken.

Nachdem die Versuchsbedingungen festgestellt waren, unter denen Strontiumoxalat unlöslich ist, wurde die volumetrische Bestimmung des Strontiums folgendermafsen ausgeführt: Die heifse Lösung des Strontiumsalzes wurde mit Ammonoxalat gefällt, sodann wurde etwa ein Fünftel bis ein Drittel des Gesamtrolumens an $85 \%$ igem Alkohol hinzugefügt. Das Gemisch blieb über Nacht stehen und die klare Flüssigkeit wurde dann über ein Asbestfilter dekantiert. Das Auswaschen geschah durch Dekantieren mit einer Mischung gleicher Volumina von Wasser und 85\% igem Alkohol. Der Niederschlag kam auf das Filter, wurde zur Entfernung des Alkohols über der Flamme getrocknet und hierauf in das vorher gleichfalls getrocknete Becherglas zurückgebracht, in dem er mit Schwefelsäure oder mit 5-10 ccm Salzsäure behandelt wurde. (Im letzteren Falle mufsten noch $0.5-1 \mathrm{~g}$ Manganosalz hinzugefügt werden.) Die Oxalsäure wurde mit Permanganat titriert. - Die nach dieser Nethode erhaltenen Resultate (Tabelle IV) sind genau.

(S. Tabelle IV, S, 149.)

Bei dem letzten Versuche, bei dem eine relativ grofse Menge Strontiumsal\% bei ziemlich geringer Konzentration vorhanden war, ist ein kleiner Verlust zu verzeichnen, der aller Wahrscheinlichkeit nach darauf zurückzufübren ist, dafs eine geringe Menge Oxalsäure durch das sich bildende Strontiumsulfat okkludiert wird. Es wäre 
Tabelle IV.

\begin{tabular}{c|c|c|c|c|c|c}
\hline $\begin{array}{c}\text { SrO, angew. } \\
\text { als } \begin{array}{c}\mathrm{Sr}\left(\mathrm{NO}_{3}\right)_{2} \\
\mathrm{~g}\end{array}\end{array}$ & $\begin{array}{c}\text { Ammon- } \\
\text { oxalat } \\
\mathrm{g}\end{array}$ & $\begin{array}{c}\text { Vol. bei der } \\
\text { Fällung } \\
\text { ccm }\end{array}$ & $\begin{array}{c}\text { Zusatz v. } \\
85 \% \text { igem } \\
\text { Alkohol }\end{array}$ & $\begin{array}{c}\text { Die während } \\
\text { der Titration } \\
\text { vorhandene } \\
\text { Säure }\end{array}$ & $\begin{array}{c}\text { Gef. } \\
\mathrm{SrO} \\
\mathrm{g}\end{array}$ & $\begin{array}{c}\text { Fehler } \\
\mathrm{g}\end{array}$ \\
\hline & & 100 & $1 / 5$ & $\mathrm{HCl}$ & 0.0973 & -0.0001 \\
0.0974 & 0.4 & 100 & $1 / 5$ & $\mathrm{HCl}$ & 0.0983 & +0.0009 \\
0.0974 & 0.4 & 100 & $1 / 5$ & $\mathrm{HCl}$ & 0.0975 & +0.0001 \\
0.0974 & 0.4 & 100 & $1 / 5$ & $\mathrm{HCl}$ & 0.0981 & +0.0007 \\
0.0974 & 0.8 & 200 & $1 / 5$ & $\mathrm{HCl}$ & 0.1943 & -0.0005 \\
0.1948 & 0.4 & 200 & $1 / 5$ & $\mathrm{HCl}$ & 0.1942 & -0.0006 \\
0.1948 & 0.8 & 100 & $1 / 3$ & $\mathrm{H}_{2} \mathrm{SO} O_{4}$ & 0.0970 & -0.0004 \\
0.0974 & 0.4 & 100 & $1 / 5$ & $\mathrm{H}_{2} \mathrm{SO}_{4}$ & 0.0977 & +0.0003 \\
0.0974 & 0.4 & 100 & $1 / 5$ & $\mathrm{H}_{2} \mathrm{SO}_{4}$ & 0.0976 & +0.0002 \\
0.0974 & 0.4 & 150 & $1 / 5$ & $\mathrm{H}_{2} \mathrm{SO}_{4}$ & 0.1938 & -0.0010 \\
0.1948 & 0.6 & & & &
\end{tabular}

demnach also zweckmälsig, die Titration bei stärkerer Verdünnung vorzunehmen, wenn man zum Ansäuern Schwefelsäure verwendet, und wenn grölsere Mengen von Strontiumoxalat vorhanden sind.

Fällung in wässeriger Lösung. Um die Vollständigkeit der Fällung von Strontiumsalzen in rein wässeriger Lösung zu prüfen, wurden $0.0974 \mathrm{~g}$ Strontiumoxyd - als Nitrat - mit Ammonoxalat ausgefällt; das Gemisch blieb über Nacht stehen, sodann wurde der Niederschlag über Asbest abgesaugt und mit Wasser, dem sein halbes Volumen an $85 \%$ igem Alkohol zugesetzt war, ausgewaschen. Das Strontiumoxalat wurde im Tiegel mit Untersatz mit einigen Tropfen Schwefelsäure behandelt und als. Sulfat zur Wägung gebracht. Es ergaben sich $0.0973 \mathrm{~g}$ Strontiumoxyd. Es ist also die Fällung des Strontiumoxalats auch in wässeriger Lösung bei Anwendung eines hinreichenden Überschusses von Ammonoxalat völlig quantitativ.

Um die für die Ausfällung des Strontiumoxalats in wässeriger Lösung erforderliche Menge Ammonoxalat festzustellen, wurden einige Versuche mit wechselnden Mengen des letzteren angestellt. Die Niederschläge blieben über Nacht in der Flüssigkeit, sodann wurde diese über Asbest dekantiert und die gefällte Substanz zwei bis dreimal mit $10-20 \mathrm{ccm}$ kaltem Wasser ausgewaschen. Die bei der Titration der Oxalsäure mit Permanganat erhaltenen Resultate zeigen, dafs es notwendig ist, eine mehrfach so grofse Menge Ammonoxalat $\mathrm{zu}$ verwenden, als theoretisch erforderlich ist, wenn man eine quantitative Abscheidung des Strontiumoxalats erzielen will. Die Versuchsdaten sind in Tabelle $\mathrm{V}$ zusammengestellt. 
Tabelle V.

\begin{tabular}{c|c|c|c|c|c}
\hline $\begin{array}{c}\text { SrO, angew. } \\
\text { als } \mathrm{Sr}\left(\mathrm{NO}_{3}\right)_{2} \\
\mathrm{~g}\end{array}$ & $\begin{array}{c}\text { Ammon- } \\
\text { oxalat } \\
\mathrm{g}\end{array}$ & $\begin{array}{c}\text { Vol. bei } \\
\text { der Fällung } \\
\mathrm{ccm}\end{array}$ & $\begin{array}{c}\text { Anwesende } \\
\text { Säure }\end{array}$ & $\begin{array}{c}\mathrm{SrO}, \\
\text { gefunden } \\
\mathrm{g}\end{array}$ & $\begin{array}{c}\text { Fehler } \\
\mathrm{g}\end{array}$ \\
\hline \hline & 0.064 & 100 & $\mathrm{H}_{2} \mathrm{SO}_{4}$ & 0.0441 & -0.0046 \\
0.0487 & 0.0768 & 100 & $\mathrm{H}_{2} \mathrm{SO}_{4}$ & 0.0465 & -0.0022 \\
0.0487 & 0.16 & 100 & $\mathrm{H}_{2} \mathrm{SO}_{4}$ & 0.0488 & -0.0001 \\
0.0974 & 0.128 & 100 & $\mathrm{H}_{2} \mathrm{SO}_{4}$ & 0.0939 & -0.0025 \\
0.0974 & 0.16 & 100 & $\mathrm{H}_{2} \mathrm{SO}_{4}$ & 0.0959 & -0.0015 \\
0.0974 & 0.32 & 100 & $\mathrm{H}_{2} \mathrm{SO}_{4}$ & 0.0975 & +0.0001 \\
\hline
\end{tabular}

Die auflösende Wirkung einer grolsen Menge Wasser auf das Strontiumoxalat wurde geprüft durch Auswaschen eines Niederschlages (mit einem Oxydgehalt von $0.0974 \mathrm{~g}$ ) mit $150 \mathrm{ccm}$ kaltem Wasser. Der als Sulfat gewogene Rückstand zeigte einen Verlust von $0.0033 \mathrm{~g}$ (auf Oxyd berechnet) und diese Quantität konnte auch aus dem Filtrat durch Zusatz von Ammonoxalat und Alkohol wieder gewonnen werden. Es ist deswegen offenbar ein allzu reichliches Nachwaschen mit Wasser zu vermeiden. Bei der quantitativen Bestimmung des Strontiums mufste deswegen, wenn die Fällung des Oxalats in wässeriger Lösung ausgeführt wurde, die Menge des Waschwassers nach Möglichkeit beschränkt werden. Es zeigte sich, dafs 30-40 ccm Wasser bei zweckmärsiger Anwendung hinreichten, das ganze Ammonsalz aus dem Niederschlage herauszuwaschen, ohne dafs von diesem eine merkliche Menge in Lösung ging.

Die Ausführung der quantitativen Bestimmung war der in alkoholischer Lösung durchaus analog; nur wurde der Alkoholzusatz fortgelassen, und die Menge des Waschwassers wurde auf ein Minimum beschränkt; da also Alkohol, der die Titration beeinflussen konnte, nicht vorhanden war, so wurde der Niederschlag vor der Titration mit Permanganat nicht getrocknet. Die Resultate sind in der folgenden Tabelle zusammengestellt.

\section{(S. Tabelle VI, S. 151.)}

Bei den unter $\mathrm{A}$ in der Tabelle VI zusammengestellten Versuchen wurde das Strontiumoxalat mit Schwefelsäure behandelt und bei $80^{\circ}$ titriert; das Volumen der Lösung betrug $200-300 \mathrm{ccm}$. Bei den Versuchen unter $B$ wurde der Niederschlag mit Chlorwasserstoffsäure behandelt und bei $35-45^{\circ}$ in einem Volumen von 
Tabelle VI.

\begin{tabular}{|c|c|c|c|c|c|}
\hline $\begin{array}{l}\mathrm{SrO} \text {, angew. } \\
\text { als } \mathrm{Sr}\left(\mathrm{NO}_{3}\right)_{2} \\
g\end{array}$ & $\begin{array}{c}\text { Ammon- } \\
\text { oxalat } \\
\mathrm{g}\end{array}$ & $\begin{array}{c}\text { Vol. bei } \\
\text { der Fällung } \\
\text { ccm }\end{array}$ & $\begin{array}{c}\text { Anwesende } \\
\text { Säure }\end{array}$ & $\begin{array}{l}\mathrm{SrO} \\
\text { gef. } \\
\mathrm{g}\end{array}$ & $\begin{array}{c}\text { Fehler } \\
\qquad \mathrm{g}\end{array}$ \\
\hline
\end{tabular}

\begin{tabular}{c|l|l|} 
& & \\
0.0974 & 0.5 & 100 \\
0.0974 & 0.5 & 100 \\
0.0974 & 0.5 & 100 \\
0.0974 & 0.5 & 100 \\
0.0974 & 0.8 & 100 \\
0.0974 & 0.8 & 100 \\
0.0974 & 1.0 & 100 \\
0.0974 & 2.0 & 100 \\
0.0974 & 2.0 & 100 \\
0.0778 & 0.5 & 100 \\
0.0778 & 0.5 & 100 \\
0.0778 & 0.5 & 100 \\
0.0778 & 0.5 & 100 \\
SrO $_{\text {angew. als }}$ & & \\
SrCl & & \\
0.0974 & & \\
0.0974 & 0.8 & 250 \\
& 2.0 & 250
\end{tabular}

A

\begin{tabular}{|l|l|r}
$\mathrm{H}_{2} \mathrm{SO}_{4}$ & 0.0966 & -0.0008 \\
$\mathrm{H}_{2} \mathrm{SO}_{4}$ & 0.0985 & +0.0011 \\
$\mathrm{H}_{2} \mathrm{SO}_{4}$ & 0.0977 & +0.0003 \\
$\mathrm{H}_{2} \mathrm{SO}_{4}$ & 0.0963 & -0.0011 \\
$\mathrm{H}_{2} \mathrm{SO}_{4}$ & 0.0981 & +0.0007 \\
$\mathrm{H}_{2} \mathrm{SO}_{4}$ & 0.0966 & -0.0008 \\
$\mathrm{H}_{2} \mathrm{SO}_{4}$ & 0.0965 & -0.0009 \\
$\mathrm{H}_{2} \mathrm{SO}_{4}$ & 0.0963 & -0.0011 \\
$\mathrm{H}_{2} \mathrm{SO}_{4}$ & 0.0970 & -0.0004 \\
$\mathrm{H}_{2} \mathrm{SO}_{4}$ & 0.0792 & +0.0014 \\
$\mathrm{H}_{2} \mathrm{SO}_{4}$ & 0.0767 & -0.0011 \\
$\mathrm{H}_{2} \mathrm{SO}_{4}$ & 0.0776 & -0.0002 \\
$\mathrm{H}_{2} \mathrm{SO}_{4}$ & 0.0776 & -0.0002 \\
& & \\
& & \\
& & \\
$\mathrm{H}_{2} \mathrm{SO}_{4}$ & 0.0973 & +0.0001 \\
$\mathrm{H}_{2} \mathrm{SO}_{4}$ & 0.0975 & -0.0001
\end{tabular}

B

\begin{tabular}{l|l|l}
0.0974 & 0.8 & 100 \\
0.0974 & 0.8 & 100 \\
0.0974 & 0.8 & 100 \\
0.0974 & 0.8 & 100 \\
0.0974 & 0.8 & 100 \\
0.0974 & 0.8 & 100
\end{tabular}

\begin{tabular}{l|l|r}
$\mathrm{HCl}$ & 0.0971 & -0.0003 \\
$\mathrm{HCl}$ & 0.0980 & +0.0006 \\
$\mathrm{HCl}$ & 0.0975 & +0.0001 \\
$\mathrm{HCl}$ & 0.0980 & +0.0006 \\
$\mathrm{HCl}$ & 0.0973 & -0.0001 \\
$\mathrm{HCl}$ & 0.0978 & +0.0004
\end{tabular}

C

\begin{tabular}{l|l|l}
0.2425 & 0.384 & 125 \\
0.2436 & 0.384 & 125 \\
0.2436 & 0.64 & 125 \\
0.2436 & 0.8 & 125 \\
0.2436 & 2.0 & 125 \\
0.2436 & 2.0 & 125
\end{tabular}

\begin{tabular}{l|l|l}
$\mathrm{H}_{2} \mathrm{SO}_{4}$ & 0.2376 & -0.0049 \\
$\mathrm{H}_{2} \mathrm{SO}_{4}$ & 0.2402 & -0.0034 \\
$\mathrm{H}_{2} \mathrm{SO}_{4}$ & 0.2411 & -0.0025 \\
$\mathrm{H}_{2} \mathrm{SO}_{4}$ & 0.2367 & -0.0069 \\
$\mathrm{H}_{2} \mathrm{SO}_{4}$ & 0.2376 & -0.0060 \\
$\mathrm{H}_{2} \mathrm{SO}_{4}$ & 0.2402 & -0.0034
\end{tabular}

D)

\begin{tabular}{l|l|l|l|l|r}
0.2436 & 0.8 & 250 & $\mathrm{H}_{2} \mathrm{SO}_{4}$ & 0.2443 & +0.0007 \\
0.2436 & 0.8 & 250 & $\mathrm{H}_{2} \mathrm{SO}_{4}$ & 0.2446 & +0.0010 \\
0.2436 & 2.0 & 250 & $\mathrm{H}_{2} \mathrm{SO}_{4}$ & 0.2440 & +0.0004 \\
0.2436 & 2.0 & 250 & $\mathrm{H}_{2} \mathrm{SO}_{4}$ & 0.2431 & -0.0005
\end{tabular}


Tabelle VI (Fortsetzung).

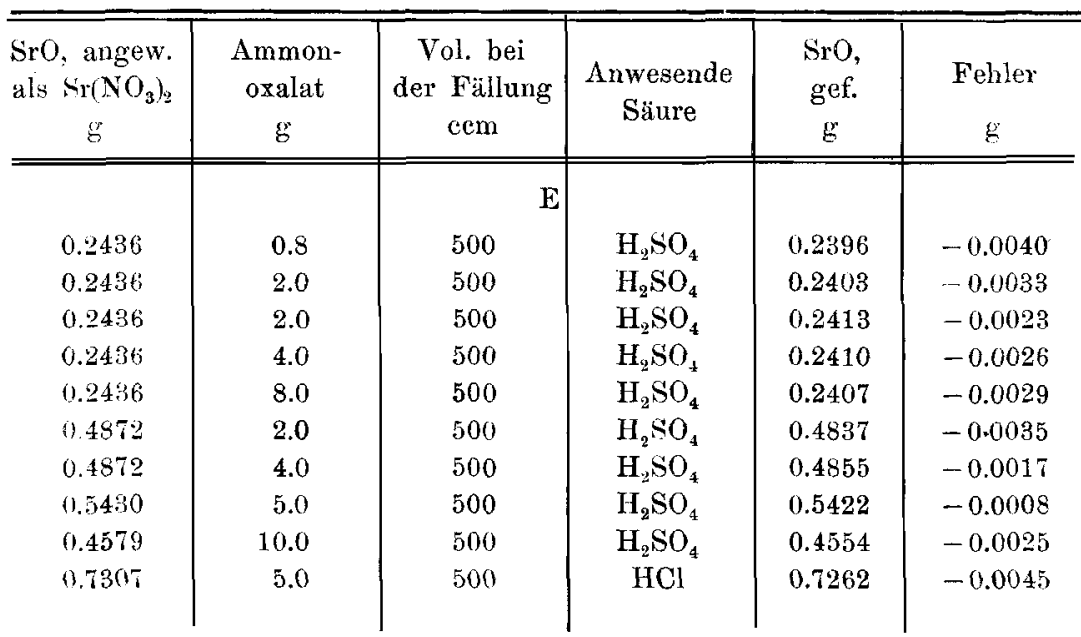

$100-200 \mathrm{ccm}$ titriert, nachdem $0.5-1.0 \mathrm{~g}$ Manganochlorid hinzugefügt waren.

Die Resultate zeigen, dafs $0.1 \mathrm{~g}$ des Strontiumsalzes, als Oxyd berechnet mit einem guten Grad von Genauigkeit als Oxalat bestimmt werden können, wenn es in $100-250 \mathrm{ccm}$ Wasser durch einen genügenden Überschuls von Ammonoxalat gefällt wird. In den Versuchen, welche in Reihe $\mathrm{C}$ wiedergegeben sind und in denen die Menge von Strontiumsalz in $125 \mathrm{ccm}$ Wasser vermehrt wird, schleicht sich ein negativer Fehler ein, der nicht durch Anwendung einer grossen Menge von Ammonoxalat vermindert werden kann. Vergrössert man jedoch die Verdünnung auf $250 \mathrm{ccm}$, wie dies in den Versuchen der Reihe $\mathrm{D}$ der Fall ist, so dafs die Bedingungen denjenigen der Reihen $\mathrm{A}$ und $\mathrm{B}$ sich nähern, dann reduzieren sich die Fehler auf ein Minimum.

Bei den unter $\mathrm{E}$ verzeichneten Versuchen, welche mit Verdünnungen von $500 \mathrm{ccm}$ ausgeführt wurden, trat eine Fehlerquelle herror, die durch die Gegenwart eines großsen Überschusses von Ammonoxalat nicht aufgehoben wird und die unabhängig ist von der angewandten Menge des Strontiumsalzes. Acht von den Wasserfiltraten und den Waschwässern der in Tabelle VI zusammengestellten Versuche wurden durch Zusatz von Alkohol auf Spuren von Strontium geprüft und stets wurde eine geringe Menge Strontium gefunden, die im Mittel $0.0010 \mathrm{~g}$ für $100 \mathrm{ccm}$ Flüssigkeit betrug. 


\section{Baryumoxalat.}

Baryumoxalat ist nach Souchay und Lenssen ${ }^{1}$ in 2590 Teilen kaltem Wasser löslich; nach BERGMANN ${ }^{2}$ ist es in Alkohol überhaupt kaum löslich. Es wurde der Versuch gemacht, Baryum durch Füllung mit Ammonoxalat in einer alkoholischen Flüssigkeit zu fallen. Dabei ergab sich, dafs in den Filtraten von Baryumoxalatniederschlägen, welche aus $0.1-0.2 \mathrm{~g}$ Baryumoxyd (als Nitrat) in $100 \mathrm{ccm}$ Flüssigkeit mit einem Gehalte von $30 \mathrm{ccm}$ abs. Alkohol gewonnen waren und über Nacht gestanden hatten, durch Schwefelsäure im Mittel nur noch etwa $0.0001 \mathrm{~g}$ Oxyd (als Sulfat) gefällt werden konnte. Die Unlöslichkeit des Baryumoxalats unter diesen Versuchsbedingungen ist demnach praktisch vollständig.

Die Bestimmung des Baryums erfolgte nun folgendermafsen: $\mathrm{Zu}$ einer Baryumsalzlösung, die $30 \%$ ihres Volumens an Alkohol

Tabelle VII.

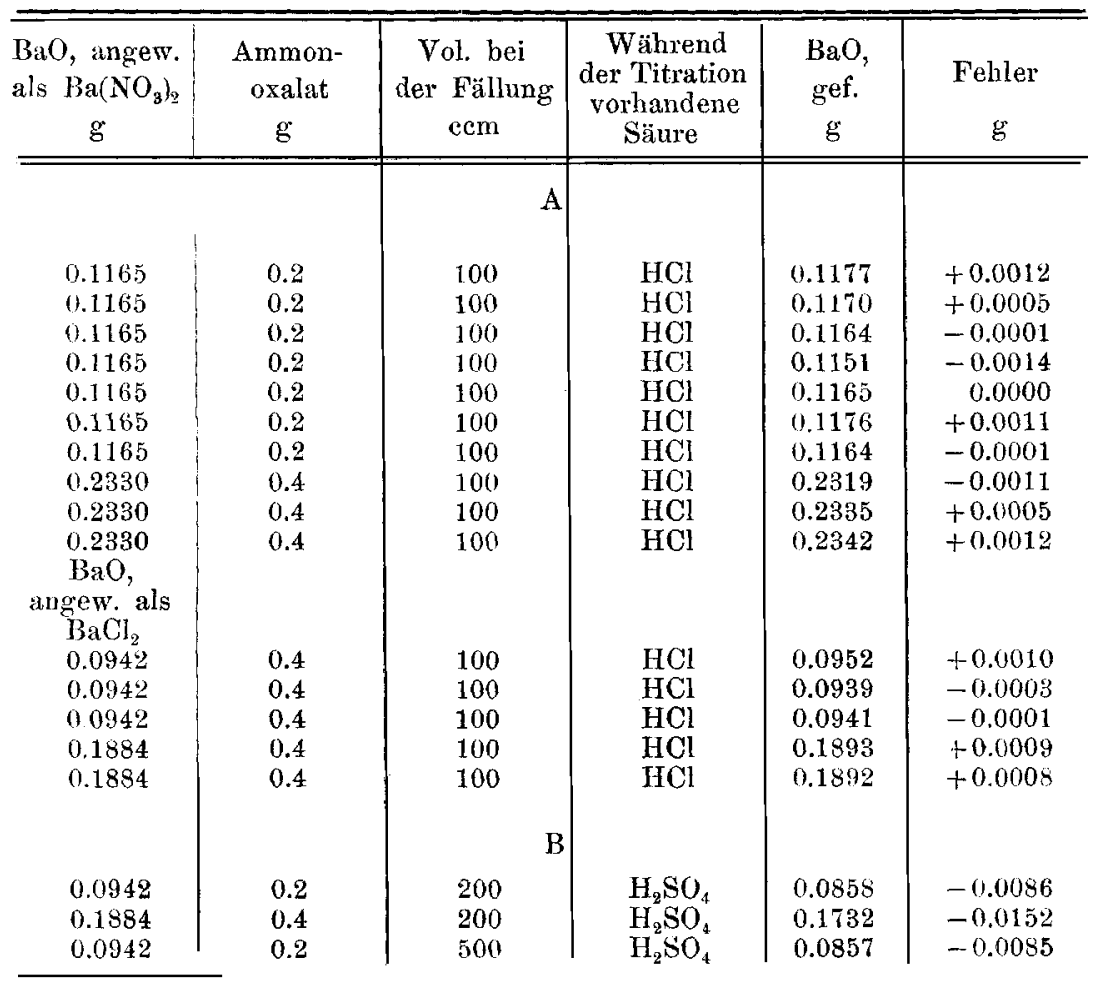

${ }^{1}$ Lieb. Ann. 90, 102.

2 Beramann's Essays I, 320. 
enthielt, wurde Ammonoxalat hinzugefügt; das Gemisch bliebt über Nacht stehen und der Niederschlag wurde über Asbest abgesaugt, nachdem er durch Dekantieren mit 100-200 $\mathrm{ccm}$ Wasser (enthaltend 30 Volumenprozente Alkohol) ausgewaschen war; hierauf wurde er über einer Flamme getrocknet zur gänzlichen Entfernung des Alkohols. Den Tiegel mit dem Niederschlag brachte man sodann in das gleichfalls vorher getrocknete Becherglas zurück, fügte $100-200 \mathrm{ccm}$ Wasser, 5-10 ccm konzentrierte Salzsäure und 0.5-1.0 g Manganochlorid hinzu und titrierte die entstandene Lösung bei $35-40^{\circ} \mathrm{C}$. mit Permanganat. Die Resultate dieser Versuche, die unter A in Tabelle VII zusammengestellt sind, zeigen, dals man Baryum als Nitrat oder als Chlorid in der beschriebenen Weise mit ziemlicher Genauigkeit bestimmen kann.

Bei den unter B verzeichneten Versuchen wurde das Baryum-

oxalat nach Zugabe der genannten Wassermenge mit Schwefelsäure behandelt. Hierbei zeigte sich ein bedeutender Verhust an Oxalsäure, der offenbar durch eine Okklusion derselben in dem entstehenden Baryumsulfat hervorgerufen wird. Die Anwendung von Schwefelsäure erscheint demnach für dieses Verfahren ausgeschlossen.

\section{Die gewichtsanalytische Bestimmung der 0xalate von Baryum und Strontium.}

Calcium kann bekanntlich nach sorgfältigem Glühen seines Oxalats als Karbonat gewogen werden, und es schien auch möglich zu sein, das Strontium als Karbonat zur Wägung zu bringen. Zur Prüfung wurden Strontiumoxalatniederschläge auf Asbest in dem mit Untersatz versehenen Filtriertiegel 2-8 Minuten lang in der Flamme eines Bunsensenbrenners geglüht und als Karbonat gewogen; in einem Falle wurde auch das so erhaltene Karbonat durch Schwefelsäure in Sulfat übergeführt und dieses zur Wägung gebracht. Die Resul-

Tabelle VIII.

\begin{tabular}{c|c|c|c}
\hline \hline Nr. & $\begin{array}{c}\text { SrO, angew. } \\
\text { als } \mathrm{Sr}\left(\mathrm{NO}_{3}\right)_{2} \\
\mathrm{~g}\end{array}$ & $\begin{array}{c}\mathrm{SrO}, \text { ber. } \\
\text { ans } \mathrm{SrCO}_{3}, \text { gef. } \\
\mathrm{g}\end{array}$ & $\begin{array}{c}\mathrm{SrO}, \text { ber. } \\
\text { aus dem gef. SrSO } \\
\mathrm{g}\end{array}$ \\
\hline \hline 1 & 0.1120 & 0.1113 & - \\
2 & 0.1120 & 0.1116 & - \\
3 & 0.2435 & 0.2425 & 0.2437
\end{tabular}


tate sind in Tabelle VIII zussammengestellt; obgleich sie einen geringen Verlust erkennen lassen, zeigen sie doch einen ziemlichen Grad von Genauigkeit.

Ebenso wurden Baryumoxalatniederschläge 5-10 Minuten geglüht und als Karbonat gewogen. Die ziemlich genauen Ergebnisse sind in Tabelle IX zusammengestellt.

Tabelle IX.

\begin{tabular}{c|c|c}
\hline \hline $\begin{array}{c}\mathrm{BaO}, \text { angew. } \\
\text { als } \mathrm{Ba}\left(\mathrm{NO}_{3}\right)_{2} \\
\mathrm{~g}\end{array}$ & $\begin{array}{c}\mathrm{BaO}, \text { gef. als } \\
\mathrm{BaCO} \\
\mathrm{g}\end{array}$ & $\begin{array}{c}\text { Differenz } \\
\mathrm{g}\end{array}$ \\
\hline 0.2912 & 0.2909 & -0.0003 \\
0.2912 & 0.2901 & -0.0011 \\
0.2912 & 0.2901 & -0.0011
\end{tabular}

Die Ergebnisse dieser Untersuchung lassen sich in der folgenden Weise zusammenfassen: Bei der Bestimmung des Calciums durch Titration des Oxalats mit Permanganat erhält man genaue Resultate, wenn man Salzsäure (unter Zusatz von Manganosalz) als Lösungsmittel benutzt. - Strontiumsalze können praktisch vollständig durch Ammonoxalat ausgefällt werden, in einer Lösung, die $1 / 5$ ihres Volumens an $85 \%$ igem Alkohol enthält; annähernd vollständig lassen sie sich auch aus rein wässeriger Lösung ausfällen, wenn die Verdünnung $250 \mathrm{ccm}$ nicht überschreitet. Das Strontiumoxalat lärst sich titrimetrisch mit Permanganat genau bestimmen, sowohl wenn man Schwefelsäure, als auch wenn man Salzsäure (unter Zusatz von Manganoxalat) zum Freimachen der Oxalsäure verwendet. Baryumsalze können praktisch vollständig durch Ammonoxalat ausgefällt werden, in einer Lösung, die ein Drittel ihres Volumens an Alkohol von $85 \%$ enthält; die Bestimmung des Baryumoxalats läfst sich in der Weise ausführen, dafs man dasselbe in Salzsäure löst und nach Zusatz von Manganosalz mit Permanganat titriert. Strontium und Baryumoxalat können durch Glühen in Karbonate übergeführt und als solche gewogen werden.

The Kent Chemical Laboratory of Yale University, New Haven, U. S. A. Bei der Redaktion eingegangen am 5. August 1901. 